\title{
Pseudorapidity dependence of multiplicity and transverse momentum fluctuations at the SPS energies
}

\author{
Daria Prokhorova for the NA61/SHINE Collaboration ${ }^{1, *}$ \\ ${ }^{1}$ Saint Petersburg State University, Saint Petersburg, Russia
}

\begin{abstract}
A search for the critical behavior of strongly interacting matter was done by studying the event-by-event fluctuations of multiplicity and transverse momentum of charged hadrons produced in inelastic $\mathrm{p}+\mathrm{p}$ collisions and central $\mathrm{Be}+\mathrm{Be}$ and $\mathrm{Ar}+\mathrm{Sc}$ collisions at the NA61/SHINE experiment. Results for energy dependence of the scaled variance of the multiplicity distribution and for two families of strongly intensive measures of multiplicity and transverse momentum fluctuations $\Delta\left[P_{T}, N\right]$ and $\Sigma\left[P_{T}, N\right]$ were presented. The study was performed in rapidity-integrated way and for different pseudorapidity regions, which corresponds to changing the rapidity averaged baryo-chemical potential and the value of temperature at the freeze-out stage. The strongly intensive measure $\Sigma\left[N_{F}, N_{B}\right]$, evaluated in rapidity separated windows, was used in the analysis of short- and long-range multiplicity correlations and is considered to be sensitive for the initial conditions of particle production such as string fragmentation and fusion.
\end{abstract}

\section{Introduction}

Fluctuation study is expected to be one of the most prominent approaches in the search for the critical behaviour of strongly interacting matter [1]. Infinite correlation length in the scaledinvariant system in the vicinity of the expected QCD critical point [2], [3] is assumed to cause divergences of distributions of measurable event quantities, e.g. particle multiplicity. In other words, expected non-monotonic behavior originated from second-order phase transition (the critical point or line) could be detected in the experiment.

The aim of strong interactions program of NA61/SHINE [4] is to study the properties of the onset of deconfinement and search for the critical point of strongly interacting matter. This study is of extreme interest now due to the plenty of experimental [5], [6] and theoretical works [7], [8], [9] investigating the structure of the phase diagram of QCD [10]. The most common scenario [11] suggests the hadron gas and quark-gluon plasma regions to be separated by a first order phase transition line at high baryo-chemical potentials and moderate temperatures. A crossover between both phases is assumed for high temperatures and low baryo-chemical potentials. The first order phase transition line then ends in a critical point. But the exact location of the critical end-point in the phase diagram is unknown. Moreover, some lattice QCD calculations suggest that there might be no critical point at all and only a crossover separates the two phases.

\footnotetext{
*e-mail: daria.prokhorova@ cern.ch
} 
Since the direct control of the freeze-out temperature and baryo-chemical potential is impossible, one can only change the initial conditions. Therefore, the main strategy of the NA61/SHINE Collaboration in this study [12] is to perform comprehensive two-dimensional scan of the phase diagram of strongly interacting matter by changing the energy and size of colliding systems. The characteristic signatures of the critical point could be observed if the freeze-out point is located close to the expected critical one.

Thuswise, if the critical point exists and could be reached within NA61/SHINE phase diagram scan program, then at some values of colliding energy and system size a region of increased fluctuations should be observed. At the top of this hill the value of the critical fluctuations is expected to be a maximum [13]. However, the challenge is to detect this properly since the critical signal could be shadowed by the trivial volume fluctuations or simply unfavourable detector effects. This led to the idea to use intensive and strongly intensive quantities as probes for the critical behaviour [14], [15].

\section{Critical probes}

In order to make proper comparison of the results obtained for different colliding systems, one should choose so-called intensive variables which are independent of the system size. Since in the vicinity of a critical point central second moments of distributions of an extensive event quantity are believed to diverge [16], the scaled variance, an intensive quantity, $\omega[N]=\frac{\left\langle N^{2}\right\rangle-\langle N\rangle^{2}}{\langle N\rangle}$ of the charged particles multiplicity $N$ was chosen for the analysis [15]. The normalization results in $\omega[N]=0$ in the absence of fluctuations of $N$ and $\omega[N]=1$ in the case of a Poisson distribution of $N[17]$. Note that $\omega[N]$ is still sensitive to fluctuations of the volume.

In particular, due to the imperfect centrality determination in A+A collisions, one should expect event-by-event volume fluctuations. Consequently, to eliminate the influence of usually poorly known distributions of the system volume, it was suggested to use strongly intensive quantities which are independent both of the volume and fluctuations of the volume within the statistical model of the ideal Boltzmann gas in the grand canonical ensemble formulation [15], [17]. Therefore, one may expect much higher sensitivity in the search of the critical point. Two families of strongly intensive variables $\Delta\left[P_{T}, N\right]$ and $\Sigma\left[P_{T}, N\right]$ were suggested [17] which are functions of two extensive event quantities: multiplicity of charged hadrons $N$ and scalar sum of their transverse momentum $P_{T}$ :

$$
\begin{gathered}
\Delta\left[P_{T}, N\right]=\frac{1}{C_{\Delta}}\left[\left\langle P_{T}\right\rangle \omega[N]-\langle N\rangle \omega\left[P_{T}\right]\right], \quad C_{\Delta}=\langle N\rangle \omega\left(p_{T}\right) \\
\Sigma\left[P_{T}, N\right]=\frac{1}{C_{\Sigma}}\left[\left\langle P_{T}\right\rangle \omega[N]+\langle N\rangle \omega\left[P_{T}\right]-2 \cdot\left(\left\langle P_{T} \cdot N\right\rangle-\langle N\rangle\left\langle P_{T}\right\rangle\right)\right], \quad C_{\Sigma}=\langle N\rangle \omega\left(p_{T}\right)
\end{gathered}
$$

The normalization of these variables can be chosen such that [17]: $\Sigma\left[P_{T}, N\right]=\Delta\left[P_{T}, N\right]=0$ in the absence of $P_{T}$ and $N$ fluctuations; $\Sigma\left[P_{T}, N\right]=\Delta\left[P_{T}, N\right]=1$ in the independent particle production model of the Boltzmann ideal gas within both grand canonical ensemble and canonical ensemble formulation.

Scaled variance and strongly intensive quantities might be sensitive to critical fluctuations as it was shown, for example, in the model of classical Van der Waals gas within grand canonical ensemble formulation [16], [18].

\section{Performed analysis}

Quantities of interest were calculated for the charged hadrons with $p_{T}<1.5 \mathrm{GeV} / c$ produced in the NA61/SHINE experimental acceptance [19]. The analysis was performed for three dif- 

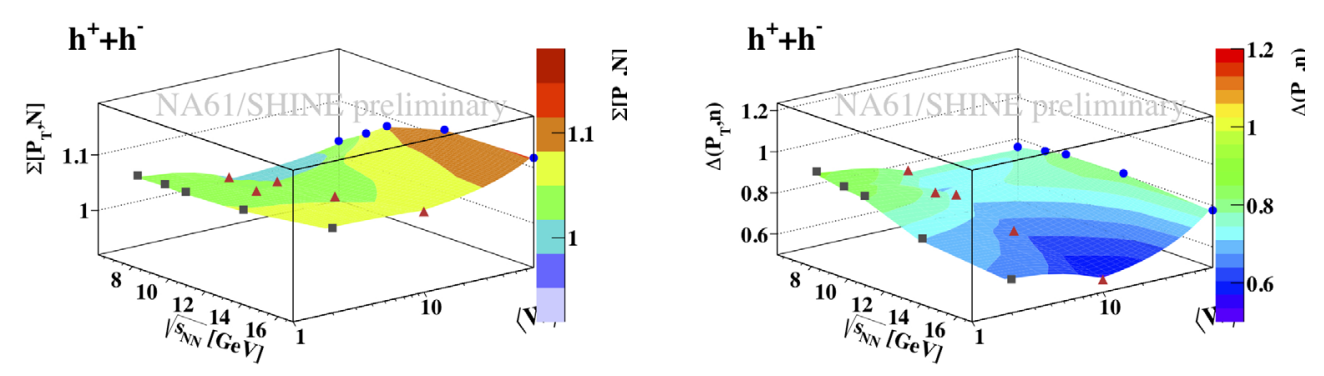

Figure 1. Experimental results [21] for $\Sigma\left[P_{T}, N\right]$ and $\Delta\left[P_{T}, N\right]$ calculated for all charged hadrons with $p_{T}<1.5 \mathrm{GeV} / c$ produced in inelastic $\mathrm{p}+\mathrm{p}$ (black squares) interactions, $5 \%$ of the most central $\mathrm{Be}+\mathrm{Be}$ (red triangles) and $\mathrm{Ar}+\mathrm{Sc}$ (blue circles) collisions. Axes are c.m.s. collision energy $\sqrt{s_{N N}}$ and mean number of wounded nucleons $\langle W\rangle[26]$

ferent systems: inelastic $\mathrm{p}+\mathrm{p}$ interactions at $20,30,40,80$ and $158 \mathrm{GeV} / \mathrm{c}$ beam momenta, as well as for $\mathrm{Be}+\mathrm{Be}$ and $\mathrm{Ar}+\mathrm{Sc}$ most central collisions at 19, 30, 40, 75 and $150 \mathrm{~A} \mathrm{GeV/c}$. Centrality was measured as a percentile of forward energy deposited by spectators in the forward hadron calorimeter PSD both in the data and in MC simulations (for the latter centrality was determined as a percentile of an energy of all final particles in the PSD kinematic acceptance [20]).

The preliminary results are presented in rapidity integrated way (Sec. 3.1) [21] and as a dependence on pseudorapidity intervals width and location (Sec. 3.2) [22], [23]. Results for $\mathrm{p}+\mathrm{p}$ and $\mathrm{Be}+\mathrm{Be}$ data were compared with the MC event generator EPOS1.99 [24] simulations calculated within the NA61/SHINE experimental acceptance efficiency [19]. For $p+p$ data the results were corrected only for off-target interactions. For all systems simulation-based corrections for other biases are in progress. The statistical uncertainties were calculated using the sub-sample method [25], and the systematic uncertainties were estimated to be smaller than $10 \%$. Further analysis of the systematic uncertainties is ongoing.

\subsection{Rapidity-integrated studies}

Experimental results for $\Sigma\left[P_{T}, N\right]$ and $\Delta\left[P_{T}, N\right]$ as functions of the collision energy and the mean number of wounded nucleons calculated with GLISSANDO model [26] were shown for all charged (Fig. 1) and negatively charged hadrons (see conference slides) produced in the interval from the zero to beam rapidity in the c.m.s. The lower edge in this case was chosen to exclude the influence of the low acceptance coverage at backward hemisphere in c.m.s., the upper bound was introduced in order to suppress possible elastic effects, which become crucial at larger pseudorapidities.

The results (Fig. 1) are shown for inelastic $\mathrm{p}+\mathrm{p}$ interactions, $5 \%$ of the most central $\mathrm{Be}+\mathrm{Be}$ and $\mathrm{Ar}+\mathrm{Sc}$ collisions. The observed structures gave no indication of the critical behavior, which led to the idea to extend the analysis by studying pseudorapidity dependence of intensive and strongly intensive quantities.

\subsection{Pseudorapidity dependence of fluctuations}

As shown in [27], the ratio of $p$ and $\bar{p}$ produced in inelastic $\mathrm{p}+\mathrm{p}$ collisions at the SPS energies changes significantly with the rapidity. Consequently, the baryo-chemical potential strongly 

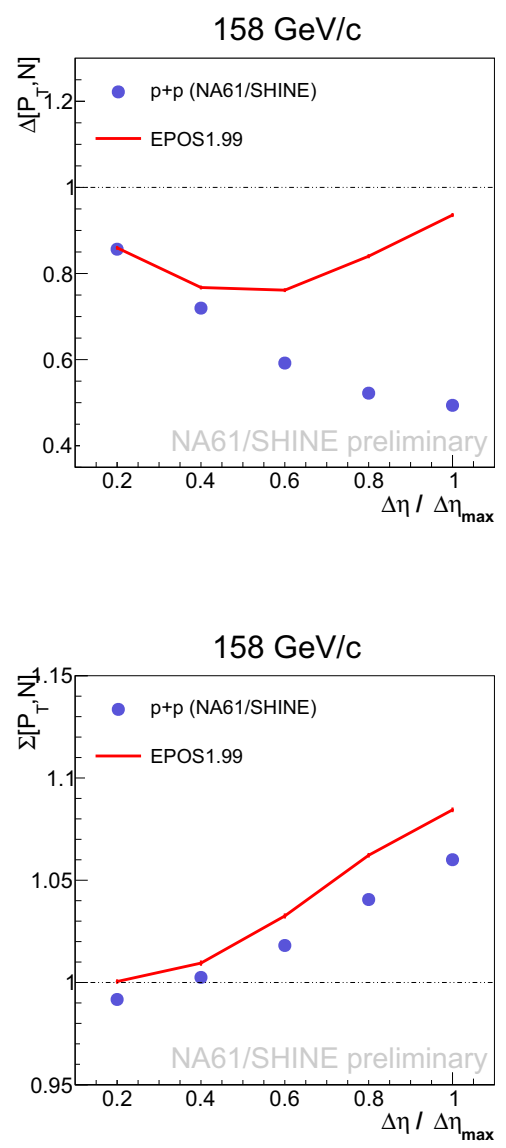
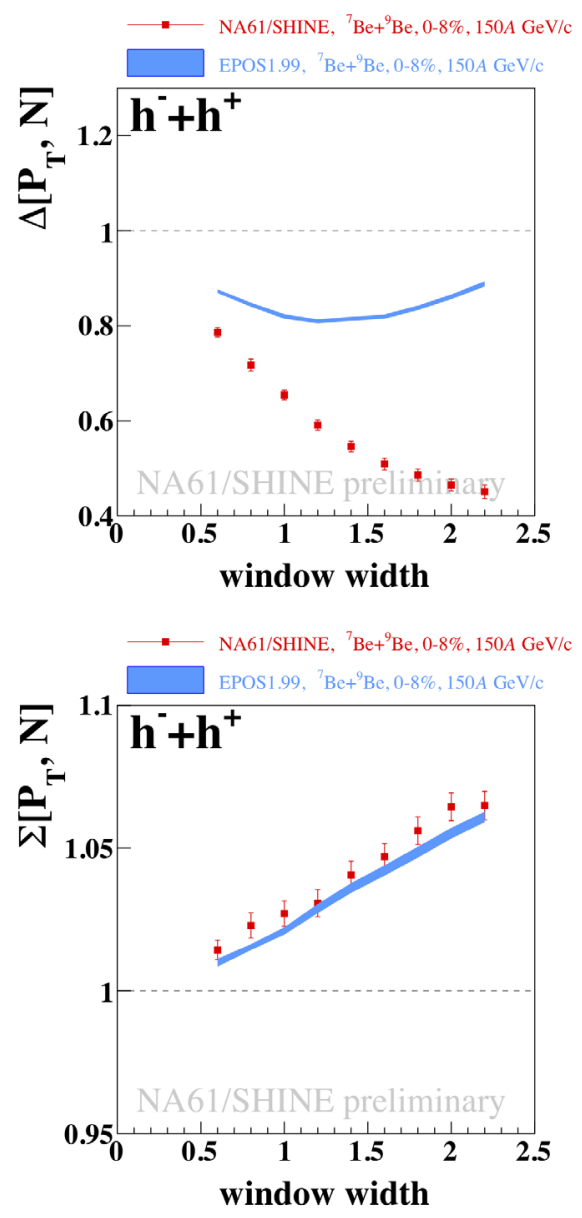

Figure 2. $\Sigma\left[P_{T}, N\right]$ and $\Delta\left[P_{T}, N\right]$ as a functions of pseudorapidity interval width for all charged hadrons with $p_{T}<1.5 \mathrm{GeV} / c$ produced in inelastic $\mathrm{p}+\mathrm{p}$ (left, window width normalized on maximal value) interactions at $158 \mathrm{GeV} / c$ [22] and $8 \%$ of the most central $\mathrm{Be}+\mathrm{Be}$ (right, window width in absolute units) collisions at $150 \mathrm{~A} \mathrm{GeV/c} \mathrm{[23].} \mathrm{Experimental} \mathrm{results} \mathrm{(dots)} \mathrm{are} \mathrm{compared} \mathrm{with} \mathrm{EPOS1.99} \mathrm{predictions}$ (lines)

depends on the pseudorapidity [28] and the choice of a pseudorapidity phase-space could probe different parts of the critical region on the phase diagram. In the previously published paper [14] the results for $p+p$ data on fluctuations integrated over the full rapidity acceptance might have averaged out possible characteristic of the critical behavior. The new advanced analysis contains two parts. The first one (Sec. 3.2.1) corresponds to the study of intensive and strongly intensive quantities in pseudorapidity intervals of the changing width, which are restricted in the reasonable phase-space of the pseudorapidity acceptance (the largest from 0 to $y_{\text {beam }}$ in the c.m.s.) within the NA61/SHINE TPC acceptance [19]. These results are relevant to different rapidity-averaged baryo-chemical potentials at the freeze-out stage [29]. The second part (Sec. 3.2.2) presents results on the pseudorapidity dependence of the strongly intensive measure $\Sigma\left[N_{F}, N_{B}\right]$ [30] calculated for the charged hadrons multiplicities $N_{F}$ and $N_{B}$ evaluated in forward $F$ and backward $B$ separated pseudorapidity intervals in $\mathrm{p}+\mathrm{p}$. 

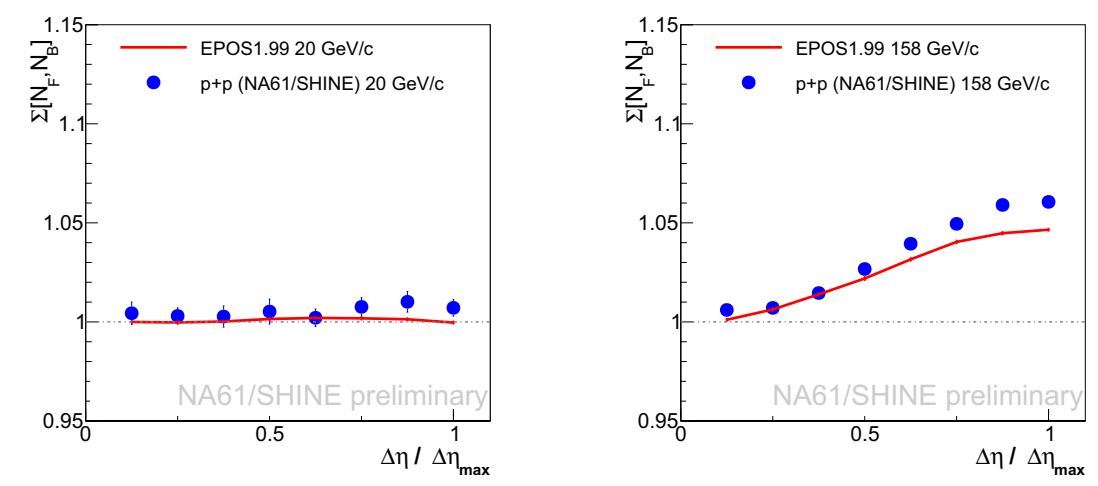

Figure 3. $\Sigma\left[N_{F}, N_{B}\right]$ for $20 \mathrm{GeV} / c$ and $158 \mathrm{GeV} / c$ beam momenta as a functions of a distance between two separated pseudorapidity intervals for all charged hadrons with $p_{T}<1.5 \mathrm{GeV} / c$ produced in inelastic $\mathrm{p}+\mathrm{p}$ [22] interactions within NA61/SHINE experimental acceptance [19]. Experimental results (dots) are compared with EPOS1.99 predictions (lines)

This quantity is supposed to be sensitive to the initial conditions of particle production such as string fragmentation, string fusion and conservation laws [31] and was used in the analysis of short- and long-range multiplicity correlations.

\subsubsection{One window analysis}

In one window analysis the results on $\omega[N], \Sigma\left[P_{T}, N\right]$ and $\Delta\left[P_{T}, N\right]$ for inelastic $\mathrm{p}+\mathrm{p}$ interactions were presented for different energies as a function of the pseudorapidity interval width [22]. The comparison of experimental values for $\Sigma\left[P_{T}, N\right]$ and $\Delta\left[P_{T}, N\right]$ with EPOS1.99 [24] results within NA61/SHINE acceptance efficiency [19] were shown for inelastic $\mathrm{p}+\mathrm{p}$ interactions and $8 \%$ of the most central Be+Be collisions (Fig. 2).

\subsubsection{Two windows analysis}

Two windows analysis results (Fig. 3) on $\Sigma\left[N_{F}, N_{B}\right]$ were presented for inelastic p+p interactions data at all beam momenta as a function of the distance between two windows and compared with the EPOS1.99 calculations within NA61/SHINE acceptance efficiency [19].

\section{Results}

NA61/SHINE conducts search for the critical point of strongly interacting matter by means of fluctuations analysis. The presented preliminary results refer to all charged hadrons with $p_{T}<1.5 \mathrm{GeV} / c$ produced in the acceptance of the NA61/SHINE experiment [19] in inelastic $\mathrm{p}+\mathrm{p}$ interactions and most central $\mathrm{Be}+\mathrm{Be}$ and $\mathrm{Ar}+\mathrm{Sc}$ collisions.

Results on system size vs. energy dependence of $\left[P_{T}, N\right]$ fluctuations (Fig. 1) show no indication of the critical point so far. Data gives (Fig. 1) $\Sigma\left[P_{T}, N\right]>1, \Delta\left[P_{T}, N\right] \leqslant 1$, which could be explained [32] by the Bose-Einstein statistics of pion gas and the negative $P_{T} / N$ vs. $N$ correlations.

The main results of pseudorapidity dependence of fluctuations revealed that fluctuation measures significantly depend on the width and the location of the pseudorapidity intervals 
(see conference slides). The value of $\omega[N]$ grows rapidly with pseudorapidity interval width, being more pronounced for higher collision energy. The value of $\Delta\left[P_{T}, N\right]$ is less than one and decreases monotonically with the increase of pseudorapidity window width (Fig. 2), with a deviation increasing with beam energy. On the other hand, the values of $\Sigma\left[P_{T}, N\right]$ are similar for all beam momenta and show the same rising tendency with increasing width of the rapidity interval (Fig. 2). The behaviour of strongly intensive quantities with a window width agrees for compared inelastic $\mathrm{p}+\mathrm{p}$ interactions and $8 \%$ most central $\mathrm{Be}+\mathrm{Be}$ collisions. Moreover, a significant discrepancy between data and EPOS1.99 calculations was observed (Fig. 2) for $\Delta\left[P_{T}, N\right]$ at all collision energies both for $\mathrm{p}+\mathrm{p}$ [22] and $\mathrm{Be}+\mathrm{Be}$ [23] collisions. The disagreement is more pronounced for the larger width of the pseudorapidity interval. However, EPOS1.99 well describes $\omega[N]$ and $\Sigma\left[P_{T}, N\right]$.

In the two windows analysis there is an increase (Fig. 3) of $\Sigma\left[N_{F}, N_{B}\right]$ value with the distance between forward and backward pseudorapidity intervals, which is more pronounced for the higher energy. The same behaviour is predicted by the model of independent quark gluon strings [31]. EPOS1.99 predictions are in a good agreement with the data.

Acknowledgments. This work is supported by the Russian Science Foundation, grant 17-72-20045.

\section{References}

[1] M.A. Stephanov, K. Rajagopal, E.V. Shuryak, Phys. Rev. Lett. 81, 4816 (1998)

[2] J. Wosiek, Acta Phys. Pol. B 19, 863 (1988)

[3] A. Bialas, R.C. Hwa, Phys. Lett. B 253, 436 (1991)

[4] N. Abgrall et al. [NA61 Coll.], JINST 9, P06005 (2014)

[5] K. Grebieszkow [NA49 Coll.], Nucl. Phys. A 830, 547C (2009)

[6] T. Anticic et al. [NA49 Collaboration], Phys. Rev. C 92, 044905 (2015)

[7] M. Asakawa, K. Yazaki, Nucl. Phys. A 504, 668 (1989)

[8] A. Barducci et al., Phys. Lett. B 231, 463 (1989)

[9] A. Bialas, R.B. Peschanski, Nucl. Phys. B 273, 703 (1986)

[10] E.S. Bowman, J.I. Kapusta, Phys. Rev. C 79, 015202 (2009)

[11] M.A. Stephanov, Prog. Theor. Phys. Suppl. 153, 139 (2004)

[12] M. Gazdzicki et al. [NA49-future Collaboration], PoS CPOD 2006, 016 (2006)

[13] M. Gazdzicki, P. Seyboth, APhysPolB 47(5), 1201 (2016)

[14] A. Aduszkiewicz et al., Eur. Phys. J. C 76, 635 (2016)

[15] M. Gorenstein, M. Gazdzicki, Phys. Rev. C 84, 014904 (2011)

[16] V. Vovchenko, D. Anchishkin, M. Gorenstein, J. PhysA: Math. Theor. 48, 305001 (2015)

[17] M. Gazdzicki, M. Gorenstein, M. Mackowiak-Pawlowska, PRC 88, 024907 (2013)

[18] V. Vovchenko, M. Gorenstein, H. Stoecker, Phys. Rev. Let. 118, 182301 (2017)

[19] https://edms.cern.ch/document/1549298/1

[20] https://edms.cern.ch/document/1867336/1

[21] E. Andronov, APhysPolB Proc. Suppl. 10, 449 (2017)

[22] D. Prokhorova, KnE Energy and Physics 3, 1:217 (2018)

[23] E. Andronov, KnE Energy and Physics 3, 1:226 (2018)

[24] K. Werner, T. Pierog, Phys. Rev. C 74, 044902 (2006)

[25] T. Czopowich [NA61/SHINE Coll.], PoS CPOD 2014, 054 (2015)

[26] W. Broniowski, M. Rybczyński. Phys. Rev. C 81, 064909 (2010) 
[27] A. Aduszkiewicz et al., Eur. Phys. J. C 77, 671 (2017)

[28] F. Becattini, J. Cleymans, J. Strumpfer, PoS CPOD 07, 012 (2007)

[29] F. Becattini, J. Manninen, M. Gazdzicki, Phys. Rev. C 73, 044905 (2006)

[30] E.V. Andronov, Theor. Math. Phys. 185(1), 1383 (2015)

[31] E. Andronov, V. Vechernin, arxiv:1808.09770 [hep-ph]

[32] M. Gorenstein, K. Grebieszkow, Phys. Rev. C 89, 034903 (2014) 